\title{
Pathogenesis of the Porcine Coronaviruses
}

\author{
DAVID J. Garwes
}

\section{GENERAL INTRODUCTION}

Of all the animal species that the coronaviruses have evolved to grow in, the pig appears to have become host to more members of the family than any other. The filterable agent identified as the cause of transmissible gastroenteritis (TGE) in American swine in the 1960s was subsequently confirmed as a coronavirus, TGEV, shortly after the family was formally identified in 1969. During the following decade, the causal agent of an encephalomyelitic disease that was associated with vomiting and wasting in swine in North America and Europe was shown to be a second coronavirus, antigenically unrelated to TGEV, named hemagglutinating encephalomyelitis virus (HEV).

A third porcine coronavirus was identified in association with a TGE-like disease of pigs in England and Europe during the 1970s. This virus, named porcine epidemic diarrhea virus (PEDV), had a pathogenesis similar to that of TGEV but was quite unrelated to either TGEV or HEV. The fourth porcine coronavirus to be identified was isolated from mild respiratory infections of European pigs that had serum antibodies to TGEV but showed no evidence of gastroenteric infection. Recent work has confirmed that this virus is closely related antigenically and genetically to TGEV but has a greatly reduced ability to grow in the intestinal mucosa. It is clear that the virus, named porcine respiratory coronavirus (PRCV), is a mutant strain of TGEV, but, as its pathogenesis is quite distinct from that of TGEV, it will be described separately.

DAVID J. GARWES - Ministry of Agriculture, Fisheries \& Food, London SW1P 3JR, England. The Coronaviridae, edited by Stuart G. Siddell, Plenum Press, New York, 1995. 


\section{TRANSMISSIBLE GASTROENTERITIS VIRUS}

\section{A. History of the Disease}

Transmissible gastroenteritis of swine was probably first described in the 1930s (Smith, 1956), although the demonstration of a viral etiology was not confirmed until the report of Doyle and Hutchings in the United States was published in 1946. They clearly showed that a filterable agent could be transmitted to piglets with the subsequent induction of a frequently fatal gastroenteritis. The growth of the science of virology following this allowed further demonstrations of the viral cause of TGE, its isolation in cell culture, and the subsequent identification of TGEV in Canada, Europe, Taiwan, and Japan (reviewed by Woode, 1969). Following the formal acceptance of the family Coronaviridae (Tyrrell et al., 1968), the causal agent of TGE was recognized as a coronavirus by Tajima (1970)

\section{B. Clinical Signs}

The severity of disease caused by TGEV is related to the age of the animal. Although pigs of all ages can become infected and sows can suffer from TGE to such an extent that agalactia occurs, it is the young piglet that usually shows the most severe clinical signs. Infection of the piglet during the first 2 weeks of life results in vomiting within 18 to $24 \mathrm{hr}$. This is usually followed by a profuse watery diarrhea that continues for several days and results in obvious dehydration and frequently death 2 to 5 days after infection. In pigs over 2 weeks of age, the initial response to infection is the same as that of the neonatal piglet, but the mortality rate drops as the body weight of the animal at the time of infection increases. This probably reflects the ability of the animal to withstand dehydration during induction of an immune response that leads to subsequent recovery.

\section{Pathology}

The usual route of infection is oral, following ingestion of contaminated material. The virus is resistant to the low $\mathrm{pH}$ of the stomach and passes to the small intestine where it infects the columnar epithelial cells covering the distal portion of the villi in the jejunum and ileum. The virus rarely colonizes the epithelium of the duodenum, supporting a hypothesis that the virus is actively taken up by the microcanalicular-vesicular system found in villus epithelium of the jejunum and ileum in the young piglet bue not in the duodenum after the second day of life (Wagner et al., 1973). The cells covering the tips of the villus are the first to be infected, and the virus then spreads down the villus while the infected cells at the tips are shed. The virus is never found in the cells at the base of the villi or in the crypt cells, and it may be that these cells are refractory to 
infection. As the infected cells are shed from the villous tips, cells migrate up from the crypt to replace them and this terminates the infection, usually 5 to 7 days after infection, as the new cells are not reinfected (Moon et al., 1976). Villous atrophy, detected as a measurable shortening of the villi, is associated with TGEV infection as with other forms of gastroenteritis (Hooper and Haelterman, 1966).

In a comparative study with attenuated and virulent strains of the virus, an apparent correlation was seen between virulence and tissue tropism. While the Purdue-115 and Gep II strains, which both induce clinical signs in week-old piglets, could be demonstrated in the enterocytes of the jejunum and ileum, Peyer's patches, and the mesenteric lymph nodes, the attenuated Nouzilly vaccine strain multiplied only in the ileum and mesenteric lymph nodes $(\mathrm{Cu}$ bero et al., 1992).

TGEV has not been detected in the cells of the large intestine, and the clinical signs appear to result from an increase in osmotic pressure in the lumen of the small intestine. The villous epithelium lost during infection carries the enymes responsible for the digestion of milk lactose, resulting in an inability to absorb the sugar (Hooper and Haelterman, 1966). Additional studies also suggested that the cells that migrate from the crypt to repopulate the denuded villi retain a secretory role, producing an abnormal level of sodium secretion into the lumen (Butler et al., 1974)) and loss of blood albumin (Prochazka et al., 1975).

TGEV can be isolated from other organs of the infected pig (Cartwright, 1967) including the kidneys and the lung. Several studies have shown that virus replication in the lung may be important for transmission and persistence of the disease (Underdahl et al., 1974; Kemeny et al., 1975). Lung infection, with TGEV antigen being detected in bronchiolar epithelial cells and alveolar cells, does not appear to be associated with respiratory disease, but may explain the emergence of the porcine respiratory coronavirus, discussed in Section III.

A study of the thymus in TGEV-infected conventional and gnotobiotic piglets suggested that, over a 4-day period, infection accelerated thymus involution, reduced thymocyte density, and resulted in hypertrophy of Hassall's bodies in the medulla (Kvachev and But, 1990).

\section{Immune Response}

Since the target cell for TGEV is the apical epithelium of the intestinal villi, host immunity requires the secretion of antibodies into the lumen of the gut. For this reason, parenteral immunization with TGEV antigens, while providing good levels of circulating serum antibodies to the virus, provides no protection against the disease. During TGE infection the immune response results in the secretion of protective antibodies into the lumen of the small intestine and this plays a part in limiting the infection in the older pig (Bohl et al., 1972a). The rapid progression of the disease to death in newborn piglets infected with TGEV probably provides insufficient time for immunity to be 
induced. It is well documented, however, that sows that have recovered from TGE can protect their offspring by passive transfer of immunity (Bay et al., 1953). Following infection of the pregnant sow, B lymphocytes secreting IgA antibodies to TGEV circulate from the gut-associated lymphoid tissue and relocate in the mammary gland. For the first 3 days after piglets are born, the colostrum secreted by the mammary gland contains antibodies derived directly from the serum. Following this, however, the only immunoglobulin present to any extent in porcine milk is secretory IgA, which is synthesized in the mammary gland. In the convalescent sow the IgA secreting cells relocated from the gut-associated lymphoid tissue can provide protective antibodies to the piglets for several weeks (Bohl et al., 1972b).

More recently, however, research findings have suggested that there may be other factors involved in the ability of milk from immune sows to protect piglets. The levels and classes of anti-TGEV antibodies were measured in milk from sows naturally infected with TGEV or immunized with the Nouzilly vaccine strain (Bernard et al., 1990). No correlation was demonstrated between the level of protection afforded and the titers of the various classes of antibodies to the virus in the colostrum and milk for the first 10 days after farrowing, although an inverse correlation was seen after 10 days.

The role of cellular immunity in recovery from TGE has not been clearly demonstrated, although studies have shown lymphocyte responses in the Peyer's patches and mesenteric lymph nodes of young piglets infected with virulent TGEV but not with attenuated virus (Welch et al., 1988). These authors suggested that this may in part explain why attenuated virus vaccines have proved to be less than fully successful for TGE. When used in the pregnant sow, vaccines have had limited success in generating lactogenic immunity, since most have failed to induce a secretory IgA response and relocation of active B lymphocytes in the mammary gland (Bohl et.al., 1975; Saif and Bohl, 1979).

\section{PORCINE RESPIRATORY CORONAVIRUS}

\section{A. History of the Disease}

During routine serological surveillance of pig herds in Great Britain, Belgium, Holland, and France in the mid-1980s, an increase in the number of herds with antibodies to TGEV was noted but with no concomitant increase in clinical enteric disease. A coronavirus, PRCV, was isolated in 1986 from respiratory tissue of affected pigs in Belgium (Pensaert et al., 1986), Great Britain (Brown and Cartwright, 1986), and subsequently from other parts of Europe and North America (Wesley et al., 1990). The virus was shown to be indistinguishable from TGEV by conventional serological tests, although monoclonal antibodies to TGEV could be used as the basis for a differential diagnostic immunoassay (Garwes et al., 1988).

By the end of the 1980s, the virus had spread to infect almost $100 \%$ of Belgium pig farms (Pensaert and Cox, 1989). It is likely that the virus is spread 
by the oronasal route and by aerosol. This is supported by data from a study with experimentally infected pigs in which airborne virus could be recovered 1 to 6 days after infection and at levels that correlated with those in the nasal mucosa (Bourgueil et al., 1992).

Several possible sources for the origin of this respiratory coronavirus have been discussed (Jestin et al., 1987). These include recombination between TGEV and another coronavirus, mutation of TGEV into a nonenteric strain, and adaptation of a TGEV-related coronavirus from another host species or its release as an attenuated TGEV vaccine. Comparative sequence analysis of the genomes from PRCV and TGEV have shown that there are high levels of homology (Britton et al., 1991; Wesley et al., 1991). The major difference between the two viruses was determined to be a 600-700 base deletion in the gene encoding the surface peplomer, suggesting that the difference in tissue tropism for the two viruses might reside in the absence of a specific cell receptor in PRCV (see Chapter 16, this volume).

\section{B. Clinical Signs}

Most observers have noted little or no sign of respiratory disease associated with infection of pigs of all ages with this virus. Experimental studies to examine the disease have given somewhat contradictory results, although differences in the PRCV isolate and the age of the pigs used may explain the findings.

O'Toole and co-workers (1989) infected colostrum-deprived piglets aged 6 to 7 days with the UK isolate of PRCV. The animals remained clinically healthy and developed specific antibodies 1 week later. Van Nieuwstadt and Pol (1989), however, used a Dutch isolate of the virus to infect specific pathogen-free pigs aged 5 weeks and recorded a severe illness in all infected animals after 1 to 2 days. The disease was characterized by anorexia, lethargy, labored respiration, and fever. Coughing was observed after 8 days and the condition improved from 10 days on, although two of the animals died of pneumonia 4 and 7 days after inoculation. Vannier (1990) noted a transient hyperthermia, mild to severe dyspnea, polypnea, and an obvious cessation of growth in colostrum-derived piglets infected by the intratracheal route.

A clinical disease, with fever, growth retardation, and lung lesions, was induced by PRCV in 6- to 8-week-old specific pathogen-free pigs and this was not exacerbated by concurrent infection with swine influenza viruses (Lanza et al., 1992).

\section{Pathology}

In the UK study, O'Toole and his co-workers (1989) examined the pathology produced by PRCV and compared it with that produced by TGEV. They found that both viruses caused a mild cranioventral bronchointerstitial pneumonia 
involving between 5 and $20 \%$ of the lung parenchyma. Both viruses were shown to replicate in the nonciliated cuboidal bronchiolar cells, and these cells then bulged into the lumen and subsequently detached. There was an accumulation of fibrin, macrophages, and cellular debris in the bronchioles, alveolar ducts, and alveoli. A minority of the infected piglets showed other pathology, including mild laryngitis, tracheitis, and rhinitis. While TGEV was identified in association with intestinal pathology, PRCV produced no intestinal lesions, although low levels of virus were isolated from the small intestine, suggesting limited replication.

The pigs that died in the study by Van Nieuwstadt and Pol (1989) were shown to have a catarrhal lobular disseminated bronchopneumonia involving all lobes of the lung. An American strain of PRCV, isolated from the intestine of a pig from a herd with endemic TGE, was capable of causing $60 \%$ consolidation of the lung 10 days after oronasal infection (Halbur et al., 1993). There was a necrotizing and proliferative bronchointerstitial pneumonia characterized by necrosis, squamous metaplasia, dysplasia, proliferation of airway epithelium, mononuclear cell infiltration of alveolar septa, mild type II pneumocyte proliferation, and lymphohistiocytic alveolar exudation. The lesions were not associated with clinical disease and were resolved by 15 days after infection.

In addition to PRCV replication in the respiratory tract, Pensaert's group demonstrated that viremia occurred and some virus reached the gastrointestinal tract (Cox et al., 1990a). There was limited replication of the virus in the jejunum, spreading to the ileum and the duodenum, in a few cells located in the subepithelial layers of the villi and crypts. Following direct inoculation into the lumen of the gut of 1-week-old piglets, PRCV replication was similarly limited to these sites and virus could be isolated from feces for several days (Cox et al., 1990b).

\section{Immune Response}

Infection of pigs with PRCV results in the production of serum antibodies that react with PRCV and with TGEV in virus neutralization, immune fluorescence, and enzyme-linked immunosorbent assay (ELISA) tests. It is likely that these antibodies confer some degree of protection on the pigs, although the mildness of the disease and the widespread occurrence of the virus make this difficult to assess. The antigenic relatedness of PRCV and TGEV suggested the possibility that seroconversion to PRCV would protect pigs against subsequent infection with TGEV. Protection against subsequent challenge with TGEV was seen in 10-week-old pigs that had been infected with PRCV by aerosol (Cox et al., 1993). However, pig herds that are known to be seropositive to PRCV have become infected with TGEV, and no protection has been afforded to nursing piglets in an experimental study (Paton and Brown, 1990). Whether this absence of cross-protection is due to critical, yet minor, antigenic differences between the two viruses or to the absence of lactogenic immunity following PRCV infection of sows has yet to be determined, although a degree of protective lactogenic immunity was claimed by Bernard et al. (1989). 


\section{PORCINE EPIDEMIC DIARRHEA VIRUS}

\section{A. History of the Disease}

A TGE-like disease was observed in young and old pigs in England at the end of 1976 and was termed "epidemic diarrhea type II" (Wood, 1977). The affected animals did not seroconvert to TGEV, however, and TGEV antigen could not be detected in the infected intestinal epithelium. Examination of gut contents from affected pigs in England (Chasey and Cartwright, 1978) and Belgium (Pensaert and Debouck, 1978) showed particles with characteristic coronavirus morphology, and the agent was recognized as a distinct coronavirus and named porcine epidemic diarrhea virus (PEDV). The disease was subsequently identified in other parts of Europe, including Czechoslovakia, Hungary, and Germany (Pensaert, 1981). Attempts to characterize the virus were hindered by its inability to replicate in cell culture until Hofmann and Wyler (1988) showed that the use of trypsin overcomes this block. The agent was subsequently shown to have physicochemical characteristics that confirmed its classification as a coronavirus (Hofmann and Wyler, 1989; Kusanagi et al., 1992)

\section{B. Clinical Signs}

The disease resembles TGE very closely and it is difficult to distinguish between them in the field. Mortality is generally restricted to piglets aged 1 week or less, but clinical signs and morbidity are frequently seen in the older animal, with diarrhea, depression, and anorexia. Experimental infection of pigs with an isolate of the PEDV from Germany, V215/78 (Witte et al., 1981), showed that a proportion of animals aged 12 weeks or older displayed severe symptoms of depression and lassitude.

\section{Pathology}

The replication of PEDV closely resembles that of TGEV, with viral antigens being detected in the epithelial cells of the villi in the small intestine shortly after infection, followed by desquamation and villous stunting. Unlike TGE, however, PEDV antigens have been detected in the epithelium of the colon. There have been no reports to date of replication in the lung.

Macroscopic lesions appear to be limited to the small intestine. The damage to the villi results in impaired intestinal function, as with TGE, but the extent of intestinal degeneration is reported to be less than that seen with TGEV.

\section{Immune Response}

Serum of pigs recovering from infection with PEDV contain antibodies to the virus detectable by immune fluorescence and virus neutralization tests. 
These antibodies do not cross-react with TGEV, and further evidence for the lack of serological relationship between the two viruses was provided by the absence of cross-protection in pigs that had recovered from infection with one virus and were subsequently infected with the other (Debouck and Pensaert, 1980). It is very likely that protection of suckling piglets against PEDV would be based on lactogenic immunity and, as discussed for TGEV, suitable vaccines have yet to be developed.

\section{HEMAGGLUTINATING ENCEPHALOMYELITIS VIRUS}

\section{A. History of the Disease}

A disease of neonatal pigs was described in Canada in 1958 in which the clinical signs were vomiting and wasting (Roe and Alexander, 1958). A similar disease, reported in Ontario the following year, developed into an acute encephalomyelitis (Alexander et al., 1959), and subsequent studies showed that the disease could be transmitted. The causal agent was not identified until 1962, however, when a virus was isolated in cell culture from piglets with encephalomyelitis (Greig et al., 1962). The virus produced syncytia in monolayer cultures of infected pig kidney cells, and the supernatant fluid from these cultures could hemagglutinate chick erythrocytes, prompting the name hemagglutinating encephalomyelitis virus (HEV).

Several years later in England, a virus was isolated in cell culture from the brain of a piglet exhibiting the vomiting and wasting disease described earlier in Canada (Cartwright et al., 1969). This virus also had the ability to produce syncytia and hemagglutinate red cells, and a serological relationship with the Canadian HEV isolate was shown, indicating that the two viruses were closely related if not identical. The virus was recognized from its morphology to be a member of the coronavirus group in 1971, and was subsequently identified in association with diseased pigs from many parts of the world (reviewed by Greig, 1981).

\section{B. Clinical Signs}

The two diseases induced by HEV, vomiting and wasting or encephalomyelitis, both become established in piglets in the first few days of their lives but are quite distinct clinically. The vomiting and wasting form starts with anorexia, depression, and inappetence. This is followed by retching and, in some piglets, vomiting of undigested milk (Lai et al., 1992). The ability to drink appears to be impaired and the piglets rapidly lose weight. There is no evidence of diarrhea, although constipation has been recorded. Three to four days after the first clinical signs are seen, infected piglets may have distended abdomens. Affected animals may die within 1 to 2 weeks but frequently survive for several weeks before dying of starvation or secondary infection. 
The encephalomyelitic form of the disease often starts with clinical signs similar to those described for the vomiting and wasting form, but the coat becomes staring, the extremities cyanotic, and signs of CNS involvement are soon manifest. These include some respiratory distress, hyperesthesia, and slight paralysis of the hind legs. In advanced stages of the disease the piglets have trouble breathing, become blind, and progress into terminal coma. The clinical signs develop over a period of about 10 days, but occasionally pigs survive and recover to show no aftereffects.

\section{Pathology}

Infection occurs via the oronasal route, and the primary site of virus replication appears to be the respiratory tract and the pharyngeal tonsils. There is little or no replication of the virus in the alimentary tract, and the vomiting produced is not related to a gastrointestinal infection as seen with TGEV and PEDV. The elegant studies of Andries and Pensaert (1980a,b) clearly demonstrated the spread of the virus by way of the peripheral nervous system to the CNS. Viral antigens were identified in the early stages of infection in the trigeminal ganglion, the inferior vagal ganglion, the superior cervical ganglion, the intestinal nervous plexuses, the solar ganglion, and the dorsal root ganglia of the lower thoracic region. Infection of the brain stem was shown to occur first in the medulla oblongata and then spread to other parts of the brain, including the cerebrum and cerebellum in later stages of infection. Virus was detected in the nervous plexus of the stomach after the incubation period, but it was not clear whether vomiting resulted from damage to these nerve plexuses or was controlled by CNS.

\section{Immune Response}

Circulating antibodies to HEV are found in serum from infected animals and these have been used to diagnose the disease by hemagglutination inhibition and virus neutralization tests. The epidemiology of HEV-induced disease suggests that herd immunity plays a part in limiting the spread of the virus (Appel et al., 1965). These authors conducted experimental infections that indicated that piglets are protected by maternal antibodies present in colostrum.

In a serological survey of pig herds in Belgium, Pensaert and co-workers (1980) found that piglets suckled by immune dams acquired neutralizing antibodies to HEV that persisted for 11 to 12 weeks. This passive immunity was replaced by active immunity between 8 to 16 weeks as the piglets became infected by virus circulating within the herd, but there were no clinical signs associated with this seroconversion, probably because the piglets were protected by the maternal antibodies. 


\section{CONCLUSIONS}

It is not obvious why the pig should provide such a suitable host for coronaviruses. Intensive rearing focuses attention on the health of a species and provides an environment that allows rapid spread of a newly emerged virus and this may provide a partial explanation. The factors that determine host species range are not clear for most virus groups, although the presence of closely related coronaviruses in pigs (TGEV), dogs (canine enteric coronavirus), and cats (feline infectious peritonitis) suggests that coronaviruses may move readily between species. HEV is antigenically related to murine, bovine, and human coronaviruses and may have evolved from one of these. The evolution of PRCV is readily explained by mutation of TGEV which is well established in pig herds in all parts of the world, but the emergence of PEDV as a pig pathogen is more difficult to explain. It is most likely that it arose from a coronavirus infecting a species of wild life in close contact with pigs. If this is so, then the conclusion may be drawn that other coronaviruses remain to be isolated from species that have not yet been studied in depth, thereby providing a reservoir from which new diseases of commercially important animals may arise.

\section{REFERENCES}

Alexander, T. J. L., Richards, W. P. C., and Roe, C. K., 1959, An encephalomyelitis of suckling pigs in Ontario, Can. J. Comp. 23:316.

Andries, K., and Pensaert, M., 1980a, Virus isolation and immunofluorescence in different organs of pigs infected with hemagglutinating encephalomyelitis virus, Am. J. Vet. Res. 41:215.

Andries, K., and Pensaert, M., 1980b, Immunofluorescence studies on the pathogenesis of hemagglutinating encephalomyelitis virus infection in pigs after oronasal inoculation, Am. J. Vet. Res. 41:1372.

Appel, M., Greig, A. S., and Corner, A. H., 1965, Encephalomyelitis of swine caused by a hemagglutinating virus. IV. Transmission studies, Res. Vet. Sci. 6:482.

Bay, W. W., Doyle, L. P., and Hutchings, L. M., 1953, Transmissible gastroenteritis in swine. A study of immunity, J. Am. Vet. Med. Assoc. 122:200.

Bernard, S., Bottreau, E., Aynaud, J. M., Have, P., and Szymansky, J., 1989, Natural infection with the porcine respiratory coronavirus induces protective lactogenic immunity against transmissible gastroenteritis, Vet. Microbiol. 21:1.

Bernard, S., Shirai, J., Lantier, I., Bottreau, E., and Aynaud, J. M., 1990, Lactogenic immunity to transmissible gastroenteritis (TGE) of swine induced by the attenuated Nouzilly strain of TGE virus: Passive protection of piglets and detection of serum and milk antibody classes by ELISA, Vet. Immunol. Immunopathol. 24:37.

Bohl, E. H., Gupta, R. K. P., McCloskey, L. W., and Saif, L. J., 1972a, Immunology of transmissible gastroenteritis, Am. J. Vet. Med. Assoc. 160:543.

Bohl, E. H., Gupta, R. K. P., Olquin, M. V. F., and Saif, L. J., 1972b, Antibody responses in serum, colostrum and milk of swine after infection or vaccination with transmissible gastroenteritis virus, Infect. Immun. 6:289.

Bohl, E. H., Frederick, G. T., and Saif, L. J., 1975, Passive immunity in transmissible gastroenteritis of swine: Intramuscular injection of pregnant swine with a modified live-virus vaccine, Am. J. Vet. Res. 36:267.

Bourgueil, E., Hutet, E., Cariolet, R., and Vannier, P., 1992, Experimental infection of pigs with the porcine respiratory coronavirus (PRCV): Measure of viral excretion, Vet. Microbiol. 31:11.

Britton, P., Mawditt, K. L., and Page, K. W., 1991, The cloning and sequencing of the virion protein 
genes of porcine respiratory coronavirus: Comparison with transmissible gastroenteritis virus genes, Virus Res. 21:181.

Brown, I., and Cartwright, S. F., 1986, New porcine coronavirus? Vet. Rec. 119:282.

Butler, D. G., Gall, D. G., Kelly, M. H., and Hamilton, J. R., 1974, Transmissible gastroenteritis: Mechanisms responsible for diarrhea in an acute enteritis in piglets, J. Clin. Invest. 53:1335.

Cartwright, S. F., 1967, Recovery of virus and copro-antibody from piglets infected experimentally with transmissible gastroenteritis, in: Proceedings of the 18th World Veterinary Congress (Paris), Organising Committee of the 18th World Veterinary Congress (eds.), 2:565, published by National Syndicate of French Veterinary Surgeons and Conseil National de l'Ordre, Paris.

Cartwright, S. F., Lucas, M., Cavill, J. P., Gush, A. F., and Blandford, T. B., 1969, Vomiting and wasting disease of piglets, Vet. Rec. 84:175.

Chasey, D., and Cartwright, S. F., 1978, Virus-like particles associated with porcine epidemic diarrhoea, Res. Vet. Sci. 25:255.

Cox, E., Hooyberghs, J., and Pensaert, M., 1990a, Sites of replication of a porcine respiratory coronavirus related to transmissible gastroenteritis, Res. Vet. Sci. 48:165.

Cox, E., Pensaert, M., Callebaut, P., and van Deun, K., 1990b, Intestinal replication of a porcine respiratory coronavirus closely related antigenically to the enteric transmissible gastroenteritis virus, Vet. Microbiol. 23:237.

Cox, E., Pensaert, M., and Callebaut, P., 1993, Intestinal protection against challenge with transmissible gastroenteritis virus of pigs immune after infection with the porcine respiratory coronavirus, Vaccine 11:267.

Cubero, M. J., Bernard, S., Leon, L., Berthon, P., and Contreras, A., 1992, Pathogenicity and antigen detection of the Nouzilly strain of transmissible gastroenteritis coronavirus in 1-week-old piglets, J. Comp. Pathol. 106:61.

Debouck, P., and Pensaert, M., 1980, Experimental infection of pigs with a new porcine enteric coronavirus, CV 777, Am. J. Vet. Res. 41:219.

Doyle, L. P., and Hutchings, L. M., 1946, A transmissible gastroenteritis in pigs, Am. J. Vet. Med. Assoc. 108:257.

Garwes, D. J., Stewart, F., Cartwright, S. F., and Brown, I., 1988, Differentiation of porcine coronavirus from transmissible gastroenteritis virus, Vet. Rec. 122:86.

Greig, A. S., 1981, Hemagglutinating encephalomyelitis, in: Diseases of Swine (A. D. Leman et al., eds.), pp. 246-253, The Iowa State University Press, Ames.

Greig, A. S., Mitchell, D., Corner, A. H., Bannister, G. L., Meads, E. B., and Julian, R. J., 1962, A hemagglutinating virus producing encephalomyelitis in baby pigs, Can. J. Comp. Med. 26:49.

Halbur, P. G., Paul, P. S., Vaughn, E. M., and Andrews, J. J., 1993, Experimental reproduction of pneumonia in gnotobiotic pigs with porcine respiratory coronavirus isolate AR310, J. Vet. Diag. Invest. 5:184.

Hofmann, M., and Wyler, R., 1988, Propagation of the virus of porcine epidemic diarrhea in cell culture, J. Clin. Microbiol. 26:2235.

Hofmann, M., and Wyler, R., 1989, Quantitation, biological and physicochemical properties of cell culture-adapted porcine epidemic diarrhea coronavirus (PEDV), Vet. Microbiol. 20:131.

Hooper, B. E., and Haelterman, E. O., 1966, Concepts of pathogenesis and passive immunity in transmissible gastroenteritis of swine, Am. J. Vet. Med. Assoc. 149:1580.

Jestin, A., Leforban, Y., and Vannier, P., 1987, Les coronavirus du porc, Rec. Med. Vet. 163:583.

Kemeny, L. J., Wiltsey, V. L., and Riley, J. L., 1975, Upper respiratory infection of lactating sows with transmissible gastroenteritis virus following contact exposure to infected piglets, Cornell Vet. 65:352.

Kusanagi, K., Kuwahara, H., Katoh, T., Nunoya, T., Ishikawa, Y., Samejima, T., and Tajima, M., 1992, Isolation and serial propagation of porcine epidemic diarrhea virus in cell cultures and partial characterisation of the isolate, $I$. Vet. Med. Sci. 54:313.

Kvachev, V. G., and But, V. I., 1990, Thymus involution in piglets with transmissible gastroenteritis, Veterinariya (Moskva) 3:29.

Lai, S. S., Ho, W. C., and Li, N. J., 1992, A preliminary report of haemagglutinating encephalomyelitis virus infections in Taiwan, J. Chinese Soc. Vet. Sci. 17:183.

Lanza, I., Brown, I. H., and Paton, D. J., 1992, Pathogenicity of concurrent infection of pigs with porcine respiratory coronavirus and swine influenza virus, Res. Vet. Sci. 53:309. 
Moon, H. W., Kemeny, L. J., and Lambert, G., 1976, Effects of epithelial cell kinetics on age dependent resistance to transmissible gastroenteritis of swine, in: Proceedings of the 4th International Pig Veterinary Society, K12, Programme Committee of the 4th International Pig Veterinary Society (eds.), published by the American Association of Swine Practitioners, Ames, Iowa.

O'Toole, D., Brown, I., Bridges, A., and Cartwright, S. F., 1989, Pathogenicity of experimental infection with "pneumotropic" porcine coronavirus, Res. Vet. Sci. 47:23.

Paton, D. J., and Brown, I. H., 1990, Sows infected in pregnancy with porcine respiratory coronavirus show no evidence of protecting their sucking piglets against transmissible gastroenteritis, Vet. Res. Commun. 14:329.

Pensaert, M. B., 1981, Porcine epidemic diarrhea, in: Diseases of Swine (A. D. Leman et al., eds.), pp. 344-346, The Iowa State University Press, Ames.

Pensaert, M., and Cox, E., 1989, Porcine respiratory coronavirus related to transmissible gastroenteritis virus, Agri-Practice 10:17.

Pensaert, M. B., and Debouck, P., 1978, A new coronavirus-like particle associated with diarrhea in swine, Arch. Virol. 58:243.

Pensaert, M., Andries, K., and Callebaut, P., 1980, A seroepizootiologic study of vomiting and wasting disease in pigs, Vet. Quart. 2:142.

Pensaert, M., Callebaut, P., and Vergote, J., 1986, Isolation of a porcine respiratory, non-enteric coronavirus related to transmissible gastroenteritis, Vet. Quart. 8:257.

Prochazka, Z., Hampl, J., Sedlacek, M., Masek, J., and Stepanek, J., 1975, Protein loss in piglets infected with transmissible gastroenteritis virus, Zentralbl. Veterinaermed. [B] 22:138.

Roe, C. K., and Alexander, T. J. L., 1958, A disease of nursing pigs previously unreported in Ontario, Can. J. Comp. Med. 22:305.

Saif, L. J., and Bohl, E. H., 1979, Passive immunity in transmissible gastroenteritis of swine: Immunoglobulin classes of milk antibodies after oral-intranasal inoculation of sows with a live low cell culture-passaged virus, Am. J. Vet. Res. 40:115.

Smith, H. C., 1956, Advances made in swine practice. IX. Transmissible gastroenteritis, Vet. Med. $51: 425$.

Tajima, M., 1970, Morphology of transmissible gastroenteritis virus of pigs, Arch. Ges. Virusforsch. 29:105.

Tyrrell, D. A. J., Almeida, J. D., Berry, D. M., Cunningham, C. H., Hamre, D., Hofstad, M. S., Mallucci, L., and McIntosh, K., 1968, Coronaviruses, Nature 220:650.

Underdahl, N. R., Mebus, C. A., Stair, E. L., Rhodes, M. B., McGill, L. D., and Twiehaus, M. J., 1974, Isolation of transmissible gastroenteritis virus from lungs of market-weight swine, Am. I. Vet. Res. 35:1209.

Vannier, P., 1990, Disorders induced by the experimental infection of pigs with the porcine respiratory coronavirus (PRCV), J. Vet. Med. Ser. B. 37:177.

Van Nieuwstadt, A. P., and Pol, J. M. A., 1989, Isolation of a TGE virus-related respiratory coronavirus causing fatal pneumonia in pigs, Vet. Rec. 124:43.

Wagner, J. E., Beamer, P. D., and Ristic, M., 1973, Electron microscopy of intestinal epithelial cells of piglets infected with a transmissible gastroenteritis virus, Can. J. Comp. Med. 37:177.

Welch, S-K. W., Saif, L. J., and Ram, S., 1988, Cell-mediated immune responses of suckling pigs inoculated with attenuated or virulent transmissible gastroenteritis virus, Am. J. Vet. Res. 49:1228.

Wesley, R. D., Woods, R. D., Hill, H. T., and Biwer, J. D., 1990, Evidence for a porcine respiratory coronavirus, antigenically similar to transmissible gastroenteritis virus, in the United States, $I$. Vet. Diag. Invest. 2:312.

Wesley, R. D., Woods, R. D., and Cheung, A. K., 1991, Genetic analysis of porcine respiratory coronavirus, an attenuated variant of transmissible gastroenteritis, $J$. Virol. 65:3369.

Witte, K. H., Prager, D., Ernst, H., and Nienhoff, H., 1981, Die epizootische virusdiarrhoe (EVD), Tierarztl. Umschau. 36:235.

Wood, E. N., 1977, An apparently new syndrome of porcine epidemic diarrhoea, Vet. Rec. 100:243. Woode, G. N., 1969, Transmissible gastroenteritis of swine, Vet. Bull. 39:239. 